\title{
Cultural Adaptations and Therapist Multicultural Competence: Two Meta-analytic Reviews
}

Timothy B. Smith

Brigham Young University, tbs@byu.edu

Alberto Soto

Brown University

Derek Griner

Brigham Young University

Melanie Domenech Rodriguez

Guillermo Bernal

Follow this and additional works at: https://scholarsarchive.byu.edu/facpub

Part of the Social and Behavioral Sciences Commons

\section{BYU ScholarsArchive Citation}

Smith, Timothy B.; Soto, Alberto; Griner, Derek; Domenech Rodriguez, Melanie; and Bernal, Guillermo, "Cultural Adaptations and Therapist Multicultural Competence: Two Meta-analytic Reviews" (2018). Faculty Publications. 3152.

https://scholarsarchive.byu.edu/facpub/3152

This Peer-Reviewed Article is brought to you for free and open access by BYU ScholarsArchive. It has been accepted for inclusion in Faculty Publications by an authorized administrator of BYU ScholarsArchive. For more information, please contact ellen_amatangelo@byu.edu. 
Soto, A., Smith, T. B., Griner, D., Domenech Rodríguez, M., \& Bernal, G. (2018). Cultural adaptations and therapist multicultural competence: Two meta-analytic reviews. Journal of Clinical Psychology: In Session, 74, 1907-1923. doi: 10.1002/jclp.22679

Cultural Adaptations and Therapist Multicultural Competence:

Two Meta-analytic Reviews

\author{
Alberto Soto, Timothy B. Smith, Derek Griner, \\ Melanie Domenech Rodríguez, and Guillermo Bernal
}

Correspondence concerning this manuscript may be directed to: Alberto Soto, Ph.D. Counseling and Psychological Services, Brown University, Providence, RI 02912 Email: soto.albert1989@gmail.com

Author Note: This article is adapted, by special permission of Oxford University Press, by the same authors in J. C. Norcross \& B. E. Wampold (Eds.) (2018), Psychotherapy relationships that work (3rd ed.). Volume 2. New York: Oxford University Press. The Interdivisional APA Task Force on Evidence-Based Psychotherapy Relationships and Responsiveness was cosponsored by the APA divisions of Psychotherapy (29) and Counseling Psychology (17). 


\begin{abstract}
Mental health treatments can be more effective when they align with the culture of the client and when therapists demonstrate multicultural competence. After providing a brief clinical example of cultural adaptations and therapist multicultural competence, we summarize relevant research findings in two meta-analyses. In the meta-analysis on the effectiveness of culturally adapted interventions, the average effect size across 99 studies was $d=.50$ (.35 after accounting for publication bias). In the second meta-analysis on 15 studies of therapist cultural competence, the results differed by rating source: Client-rated measures of therapist cultural competence correlated strongly $(r=.38)$ with treatment outcomes but therapists' self-rated competency did not $(r=.06)$. We describe patient considerations and limitations of the research. We conclude with research-supported therapeutic practices that help clients benefit from cultural adaptations and from therapists they perceive as culturally competent.
\end{abstract}

Keywords: multicultural competencies, cultural adaptations, multicultural psychotherapy, metaanalysis, treatment adaptations 
Cultural Adaptations and Therapist Multicultural Competence:

Two Meta-analytic Reviews

A client's cultural experiences and background impact the client-therapist relationship, selection of treatments, the formation of the alliance, and the outcome of psychotherapy (Casas, Suzuki, Alexander, \& Jackson, 2016; Vasquez, 2007). It is crucial that mental health treatments account for client culture (e.g., La Roche, 2012; Yeh, Parham, Gallardo, \& Trimble, 2011). Explicitly attending to culture improves the complex processes and multifaceted outcomes inherent in psychotherapy (La Roche \& Christopher, 2008). Over the past four decades, major advances in scholarship have raised awareness of cultural factors in psychotherapy, with particular emphasis on therapist cultural competencies (Imel et al., 2011; Tao, Owen, Pace, \& Imel, 2015) and culturally adapted treatments (Benish, Quintana, \& Wampold, 2011; Hall, Ibaraki, Huang, Marti, \& Stice, 2016). This article addresses both cultural adaptations to treatments and therapist cultural competence with clients from diverse racial and ethnic groups. Both topics are essential and provide frameworks for how to be with a client, as well as what to do to align psychotherapy with clients' cultures.

\section{Definitions}

Cultural adaptations of mental health treatments involve systematic modifications "to consider language, culture, and context in such a way that it is compatible with the client's cultural patterns, meanings, and values" (Bernal, Jiménez-Chafey, \& Domenech Rodríguez, 2009, p. 362; see also Cardemil, 2010). For instance, cultural adaptations might include a holistic/spiritual conceptualization of wellness and engagement in cultural rituals with North American Indians and Alaskan Natives (King, Trimble, Morse, \& Thomas, 2014).

Treatment adaptations meaningfully aligned with client culture (La Roche \& Lustig, 
2010, 2013) include multiple components, such as treatment goals or methods. Bernal, Bonilla, and Bellido (1995) nominate eight domains of psychotherapy that are amenable to cultural adaptations: language, persons, metaphors, content, concepts, goals, methods, and context. For example, language adaptations include oral and written adaptations to communication, utilizing preferred languages/dialects, code switching, or culture-specific jargon. Content adaptations address cultural customs or values, such as familismo (importance of strong family connections) when working with Latinx clients. Thus, culturally adapted therapy considers multiple factors not typically addressed in traditional psychotherapy (Smith \& Trimble, 2016).

Whereas cultural adaptations involve modifications to the treatment itself, cultural competence refers to therapists' abilities to actively engage and work effectively with diverse clientele (Domenech Rodríguez \& Bernal, 2012). Multicultural competencies are frequently operationalized in a tripartite model consisting of awareness, knowledge, and skills (Arredondo et al., 1996; Sue, Arredondo, \& McDavis, 1992).

Cultural awareness refers to therapists' ability to recognize the cultural background of themselves and their clients, including assumptions and biases that influence the process of psychotherapy with diverse clientele. Cultural knowledge refers to therapist understanding of specific cultural groups, their norms and unique experiences (e.g., how the tenets of Islam and the traumatic experiences of civil war impact a refugee family from Syria), as well as the ways in which historical forms of oppression (e.g., colonialism) continue to impact the assumptions of psychotherapy. Finally, cultural skills refer to therapists' ability to actively engage diverse clientele and to modify assessment or treatment methods to better match the cultural needs of clients. Cultural competence has become part of ethical and professional standards of psychology (e.g., APA, 2017a, 2017b) and other mental health professions (e.g., ACA, 2014; NASP, 2010). 


\section{Measures}

\section{Cultural Adaptations}

Researchers typically provide a brief narrative explanation of cultural adaptations implemented in the study, without any quantification of the extent or quality of the adaptations. However, scholars distinguish between adaptations developed prior to clinical implementation for a broad population, such as an intervention for African American clients, and local adaptations made with a specific population, such as African American clients with diabetes in a particular community (Barrera, Berkel, \& Castro, 2017).

Our prior meta-analyses (Griner \& Smith, 2006; Smith, Domenech Rodríguez, \& Bernal, 2011; Smith \& Trimble, 2016) have quantified the extent of cultural adaptations (e.g., Bernal et al., 1995). Other meta-analyses have evaluated specific types of adaptations (e.g., language match), adaptation to explanatory model of illness (illness myth adaptation), adherence to specific model of adaptation, or overall quality of adaptations (Benish et al., 2011; Cabral \& Smith, 2011; Degnan et al., 2017; Hall et al., 2016; Huey \& Polo, 2008).

\section{Cultural Competence}

Several therapist self-report measures of therapist cultural competence exist. The four measures used most often in research are the Multicultural Counseling Inventory (MCI; Sodowsky, Taffe, Gutkin, \& Wise, 1994), the Cross-Cultural Counseling Inventory-Revised (CCCI-R; LaFromboise, Coleman, \& Hernandez, 1991), the Multicultural Counseling Awareness Scale (MCAS; Ponterotto, Gretchen, Utsey, Rieger, \& Austin, 2002), and the Multicultural Awareness Knowledge Skills Scale (MAKSS; Kim, Cartwright, Asay, \& D’Andrea, 2003).

Reviews of these instruments have been largely favorable (e.g., Ponterotto, Rieger, 
Barrett, \& Sparks, 1994; Pope-Davis \& Dings, 1994, 1995). All four of the instruments were found to be sensitive to therapist change as a result of training (Ponterotto \& Alexander, 1996). A reliability generalization study (Dunn, Smith, \& Montoya, 2006) of measures of cultural competence found acceptable psychometric evidence of reliability across 89 studies; however, these authors pointed to inadequate evidence of divergent and construct validity.

At the same time, these measures of therapist cultural competence manifest limitations. First, participant responses may be impacted by social desirability (e.g., Constantine \& Ladany, 2000), although one review suggested this impact may be relatively small (Dunn et al., 2006).

Second, the self-report versions actually measure multicultural therapy self-efficacy, not demonstrated ability to counsel diverse populations (Constantine et al., 2000, 2002). Third, therapist self-report measures are unreliably related to observer ratings (e.g., Worthington et al., 2000) or to clients' evaluations of therapists' cultural competence (Smith \& Trimble, 2016).

Finally, psychometric concerns such as low concurrent validity and high correlations between subscales have "raised questions about the development and definition of the constructs being measured" (Kocarek, Talbot, Batka, \& Anderson, 2001, p. 494).

Scholars have also examined therapist variability among client outcomes between diverse and White clients as a function of either therapists' general competence or cultural competence. Research has shown that general competencies and cultural competencies can be distinguished (Imel et al., 2011). Therapist effectiveness can differ across client race or ethnicity (Hayes, Owen, \& Bieschke, 2014). Demonstrable improvement of culturally diverse clientele provides evidence of therapist cultural competence (Hayes et al., 2016).

\section{Clinical Example}

"Nolan" is a 28-year-old African American male from Minnesota. He is in a committed 
relationship and identifies as heterosexual. Spirituality is an important part of his life, but he is not currently active with his church. Nolan was raised by his mother, who has struggled with substance dependence, as has his younger brother. He presents to psychotherapy with worsening depression after discovering that his brother and mother were using drugs together, an experience that elicited deep feelings of betrayal and isolation. In addition, Nolan states that the widely publicized shooting of Philando Castile, and the resultant lack of legal conviction of the involved police officer, have increased his feelings of depression and hopelessness.

During the course of treatment, both the context and content of therapy were adapted to Nolan's experience as an African American man. His therapist engaged in open discussion of topics such as police brutality, recent political changes, and Black Lives Matter as they related to Nolan's feelings of distress and isolation. Nolan and his therapist also discussed Afrocentric literature (e.g., Asante, 2009), and the therapist used concepts and principles of NTU psychotherapy (Phillips, 1990), an Afrocentric approach to psychotherapy based on cultural and spiritual values. Using that culturally adapted treatment, the therapist helped Nolan explore his family relationships, his spirituality, his connection to ancestors, and work toward the five core NTU principles: harmony, balance, interconnectedness, cultural awareness, and authenticity.

When working with Nolan, the therapist sought cultural competence by remaining aware of personal biases, increasing understanding of Nolan's circumstances, and interacting with him in ways that met his needs and experiences. Striving for the awareness, knowledge, and skills of cultural competence, the therapist worked through personal beliefs (e.g., victim blaming) that conflicted with Nolan's experiences. The therapist actively solicited feedback, such as learning how the African American community reacted to the death of Philando Castile in Minneapolis. Tempering the expectation that Nolan would have to explain everything, the therapist also sought 
out relevant research and readings, in addition to demonstrating cultural humility when referencing salient information (e.g., “Can you help me understand what I am missing?”). Thus, the therapist's attention to cultural competency fostered the working alliance with Nolan and allowed for the culturally adapted interventions, including effectively discussing the broader context of racism relevant to Nolan and his family's circumstances. Multicultural competencies guided the processes for working with Nolan's symptoms of depression in the context of his cultural experiences.

\section{Methods of the Meta-Analytic Reviews}

\section{Inclusion and Exclusion Criteria}

For the first meta-analysis of cultural adaptations, we included only studies that used experimental and quasi-experimental research designs that evaluated mental health treatments that were culturally adapted based on considerations for people of color in the United States or Canada. For the second meta-analysis of cultural competencies, we included studies that examined the correlation between therapist multicultural competencies and client outcomes (clinical improvement and premature dropout vs. completion). The meta-analysis of cultural adaptations included only experimental/quasi-experimental studies, while the meta-analysis of multicultural competencies included cross-sectional and longitudinal evaluations of the association among variables.

The specific criteria for inclusion were as follows: (1) Studies of people of color with less than $10 \%$ non-Latinx Whites or European Americans; (2) data evaluated psychological treatments; (3) primary outcomes were psychological in nature (e.g., anxiety, OCD), excluding substance-use outcomes; (4) manuscripts were in English or Spanish on studies conducted in the United States or Canada; (5) effect sizes could be extracted for the effectiveness or efficacy of 
culturally adapted interventions (for the first meta-analysis) or for the relation between multicultural competencies and client outcomes (for the second meta-analysis).

\section{Information Sources and Search Strategies}

To locate relevant published and unpublished studies through May 2017, we searched PsycINFO, EMBASE, Academic Search Premier, and ProQuest Digital Dissertations. We used dozens of synonymous search words and phrases, such as racial/ethnic/culture specific/sensitive/adapted, that were cross-referenced with word roots and phrases specific to mental health treatments. Additionally, we searched the references of all articles containing data to identify additional studies. Team members further searched for articles citing the retrieved studies. Solicitations for unpublished manuscripts were posted on professional listservs.

\section{Data Collection Processes}

Our research team, consisting of two university faculty members, two graduate students, and five undergraduate students, received extensive training on coding procedures. Two pairs of raters coded each article (four coders per article). The inter-rater agreement of initial coding decisions for the first meta-analysis on cultural adaptations averaged .95 using intraclass correlation coefficients for continuous variables and .70 using Cohen's kappa for categorical variables. For the second meta-analysis on multicultural competence, the coefficients were .89 and .74, respectively. Discrepancies were resolved through discussion and mutual agreement. Coders extracted effect size data and information about possible moderating variables, including participant characteristics (e.g., age, race) and study characteristics (e.g., sample size). The meta-analysis on cultural adaptations coded for specific types of cultural adaptations, following the model proposed by Bernal and colleagues (1995). The meta-analysis on cultural competencies coded study and participant characteristics, as well as the source of therapist 
cultural competency rating (e.g., self, client, or observer-rated) and reliability coefficients of the cultural competence scale. The moderator variables for culturally adapted treatments are listed in Table 1, and those for therapist cultural competency are listed in Table 3.

\section{Data Analyses}

We used the Meta-Analysis Calculator (Lyons \& Morris, 2017) to transform statistical estimates derived from a variety of metrics (e.g., $F$ and $p$ values) to Cohen's $d$ or Pearson's $r$. For the first meta-analysis of cultural adaptations, positive effect sizes indicated superior efficacy of adapted treatments while negative effect sizes indicated that the non-adapted interventions were superior. For the second meta-analysis of multicultural competencies, positive effect sizes indicated better client outcomes associated with therapist cultural competence, whereas negative effect sizes indicated poorer client outcomes were associated with multicultural competence. This correlational data was transformed to Fisher's $z$, to allow for proper aggregation and then subsequently transformed back to Pearson's $r$ for reasons of interpretation.

For both meta-analyses, effect sizes were aggregated within studies, with one effect size per study in the overall analyses. When studies contained multiple effect sizes (e.g., depression and anxiety outcomes), the multiple effect sizes were averaged, weighted by the standard error or number of participants. Data analyses were conducted in STATA using random effects models.

\section{Meta-analysis of Cultural Adaptations to Treatments}

\section{The Studies}

We located 99 studies evaluating a cultural adaptation to a mental health treatment that met our inclusion criteria, including 21 additional studies beyond those reported in a prior metaanalysis (Smith \& Trimble, 2016). Most of the studies had been published (82\%) and had appeared after the year 2000 (66\%). The average client age was 26.8 years, but clients were 
predominantly children/adolescents $(46 \%)$ or middle-aged adults $(40 \%)$, which reflected a trend for either prevention-oriented treatments for at-risk groups or treatments in community mental health clinics. Accounting for that trend, we analyzed differences between these two groups.

Overall, the studies contained data from 13,813 individuals, with a median of 60 participants and with an average of $63 \%$ females. Asian American (39\%) and Hispanic/Latinx American (40\%) clients were the most commonly evaluated racial and ethnic groups. African Americans (15\%) and Native American (5\%) clients were evaluated less frequently.

The types of cultural adaptations reported in individual studies varied substantially, both in terms of the number of adaptations and in terms of the specific types of adaptations. Some studies clearly followed existing guidelines available in the professional literature (e.g., Bernal et al., 1995), whereas others provided minimal descriptions and/or had restricted adaptations that focused on only a few aspects of culture. Across all studies the three most frequent adaptations were: providing treatment in the preferred language of the client $(75 \%)$, explicit mention of cultural content/values in treatment (75\%), and matching clients with therapists of similar racial/ethnic background (55\%). On average, studies reported utilizing 3.8 out of the 8 components proposed by Bernal and colleagues (1995). Only 18 studies (18\%) involved more than 5 of the 8 components. Thus, researchers typically made several attempts to culturally adapt treatments; a small minority made comprehensive adaptations.

Separate analyses were conducted for 76 studies with experimental designs and 23 with quasi-experimental designs. We also analyzed 45 studies with no-treatment controls separately from 53 studies in which cultural adaptations were compared with a non-adapted treatment.

\section{Overall Results}

Across all 99 studies examining a culturally adapted mental health treatment $(13,813$ total 
participants), the random effects weighted effect size was $d=0.50$ (se $=.04,95 \% \mathrm{CI}=0.42$ to $0.58, p<.0001)$, a moderately strong effect. The heterogeneity of the findings was high $\left(I^{2}=\right.$ $71.4,95 \% \mathrm{CI}=65$ to $\left.77 ; Q_{(98)}=342, p<.0001\right)$, indicating that the results were inconsistent across studies (see Figure 1). Thus, further analyses were conducted in order to account for the inconsistency across studies.

\section{Publication Bias}

When findings are obtained that are non-significant (null findings), those results are less likely to be published or may not exist and are therefore unable to be located in a literature search and included in the meta-analysis. This inability to locate unpublished studies can result in publication bias. In this meta-analytic review, publication bias appeared to influence the overall findings. Figure 1 depicts asymmetric data (few studies appear in the bottom left), reflecting few studies with negative or null findings. The distribution strongly suggests publication bias in the available literature.

Published studies had higher average effect sizes than unpublished studies $(d=0.54$ versus $d=0.31, p=.04)$. Egger's regression test and Begg's test for small-study effects were both statistically significant $(p<.001)$, providing further evidence of publication bias. Furthermore, the trim and fill method (Duval \& Tweedie, 2000) identified 25 "missing" studies in the distribution. When those hypothetically missing data were accounted for, the resulting omnibus effect size was reduced to $d=0.35$ (95\% CI $=0.27$ to 0.43$)$. Thus, the results presented in the previous section represent liberal estimates likely impacted by publication bias. The value of $d=0.35$ provides a more conservative estimate accounting for publication bias.

\section{Moderators}

Given the heterogeneity of the overall findings, we conducted a series of random-effects 
weighted analyses of variance and meta-regressions to examine variables that could have accounted for the variance in effect size estimates. We first examined study, treatment, and participant characteristics separately (Table 1). As an additional step, we included these variables simultaneously in a meta-regression model (Table 2).

Moderation by study characteristics. Studies using random assignment (i.e., experimental designs) produced larger effects than those utilizing non-random assignment of clients to treatment conditions ( $d=0.56$ vs. $d=0.31, p=.003$; see Table 1$)$. This finding was unexpected, as experimental designs typically account for multiple potential confounds and typically result in more conservative effect sizes, compared to designs in which confounds are uncontrolled. Additionally, 45 studies comparing outcomes of treatment groups to those of notreatment control groups (i.e., clients on a waiting list; $d=0.53$ ) yielded similar findings to the 53 studies comparing outcomes in the experimental group to outcomes of clients receiving some kind of treatment (e.g., treatment as usual; $d=0.47, p>.10$ ). It would be expected that studies comparing adapted treatments to no treatment would result in larger effect sizes, but the difference observed was small, an average of $d=0.06$.

Results also varied significantly across four types of outcome assessment $(p=.002)$. As indicated in Table 1, studies evaluating general/multi-dimensional well-being averaged $d=0.23$, and studies evaluating general/multi-dimensional mental health symptoms averaged $d=0.26$. However, studies evaluating specific mental health symptoms (e.g., depression) averaged $d=$ 0.55, and studies evaluating specific behavioral outcomes (e.g., aggression) averaged $d=0.58$. These two sets of evaluations, general versus specific, yielded quite different results.

We also found that effect sizes differed as a function of the year of study publication. The random effects weighted correlation of study year with effect size was .20 ( $p=.02)$. All of the 
studies with effect sizes greater than 1.50 appeared during the years 2004 to 2014 . There were no studies prior to the year 1999 that reported effect sizes larger than 1.0. This trend in the data could, in part, be explained by improvements to the culturally adapted treatments: We observed a moderate correlation of $.24(p=.04)$ between an estimate of adaptation quality and year of study publication. This trend of larger effect sizes over time was not attributable to an estimate of publication bias (i.e., inverse of the number of participants or the standard error).

Moderation by treatment characteristics. After restricting analyses to studies using a comparison group that received a treatment (rather than a no-treatment control), mental health treatments produced smaller effects $(d=0.41)$ than programs targeting problem behaviors such as aggression $(d=0.68 ; p=.01)$. However, average effect sizes were similar across individual, group, and family treatments $(p>.10)$.

Examining specific cultural adaptations indicated that treatments conducted in the preferred language of the client (when the client did not speak English as a native language) produced larger effects than those that did not explicitly report conducting treatment in the preferred language of the client $(d=0.59$ versus $.35, p=.03)$. Similarly, studies that provided written assessments translated to clients' preferred language produced much larger effect sizes than those that did not $(d=0.66$ versus $.28, p<.001)$. When clinical goals were explicitly based on client cultural values, effect sizes were much larger than when no such information was provided $(d=0.69$ versus $.46, p=.03)$. Treatments involving cultural metaphors were more effective than those that did not mention that particular cultural adaptation $(d=0.61$ versus $.43, p$ $=.02$ ). Additionally, when the treatment methods (e.g., performing cultural rituals, consulting with family members) were culturally adapted, the studies yielded larger effect sizes than those not explicitly modifying treatment methods $(d=0.58$ versus $0.42, p=.04)$. Overall, the more 
cultural adaptations reported in the study, the larger the effect size $(r=.21, p=.01)$.

Moderation by participant characteristics. Effect sizes were not moderated by participant gender. Similarly, the percentage of clients and therapists from various racial groups were unrelated to effect size magnitude. However, experimental studies that contained all participants of the same race produced larger effect sizes than studies in which clients of various races received the same culturally adapted treatment $(d=0.63$ versus $0.29, p=.01)$.

Clinical samples $(d=0.61)$ produced stronger benefits from treatment than community and at-risk samples $(d=0.44, p=.04)$. This pattern also remained after restricting analyses to experimental designs. Twenty-seven treatments for clinical populations yielded an effect size of $d=0.80$, and 49 interventions for community and at-risk samples yielded an average effect size of $d=0.48(p=.003)$. Thus, the type of client sample moderated the overall findings.

Effect sizes were also moderated by participant age $(r=.25, p=.004)$. Examination of the associated scatterplot confirmed that studies with participants older than 40 years yielded larger effect sizes. Experimental studies with at-risk children and adolescents tended to produce smaller effects $(d=0.36)$ than those with adult clinical samples $(d=0.70 ; Q=9.1, p=.003)$.

Meta-regression of moderating variables. The previous analyses in this section identified several variables that explained systematic differences in effect sizes. Some of these variables, such as client age and clinical status, clearly overlapped. We therefore sought to ascertain which of the several moderating variables would remain predictors of treatment effectiveness in the presence of the others.

A meta-regression including only the 93 studies reporting participant age explained $35.4 \%$ of the variance in effect sizes $(p<.001)$, with the results depicted in Table 2 . All variables except two contributed at least $1 \%$ of variance to the model; year of study publication and racial 
or ethnic homogeneity were weak predictors in the presence of the other variables. An indirect estimate of publication bias (the inverse of study sample size; Peters et al., 2006) remained the strongest predictor, explaining $6.6 \%$ of effect size variance. The use of specific versus general assessments and random assignment to treatments also explained substantial amounts of variance (6.1\% and $4.6 \%$, respectively). In the presence of the other variables, the number of reported cultural adaptations remained a statistically significant predictor; the efficacy of a treatment increased as more cultural adaptations were incorporated. Client age and at-risk versus clinical status were not significant when examined with the other variables.

A separate meta-regression was conducted to examine which cultural adaptations were most predictive of positive client outcomes. Indicators of the eight components of the ecological validity model (Bernal et al., 1995) explained $14.5 \%$ of the variance in effect sizes $(p<.01)$. The two types of cultural adaptations that remained statistically significant were (a) explicitly basing treatment on the client's goals, informed by cultural values (standardized beta $=0.24, p=.01$ ) and (b) providing treatment in clients' preferred language (standardized beta $=0.18, p<.05$ ).

\section{Meta-analysis of Therapist Multicultural Competence}

\section{The Studies}

We identified 15 studies containing data on 2,640 clients' experiences in mental health treatments as a function of their therapists' level of multicultural competence. Nine studies $(60 \%)$ involved clients receiving individual psychotherapy and seven studies evaluated clients participating in various formats (individual and group treatments).

The majority of studies (73\%) appeared since the year 2010, including many unpublished doctoral dissertations (40\%). All studies used convenience samples, with most (62\%) using university/college students as participants. Studies averaged 176 clients, with one study having 
more than 500 participants. Most often clients were either African American (41\%) or Hispanic/Latinx American (32\%), with limited research investigating other racial or ethnic groups. Nine studies involved cross-sectional designs, and six studies evaluated changes over time. In this meta-analysis, two outcomes were considered: client participation in treatment (premature termination vs. completion), evaluated in four studies; and treatment outcomes, evaluated in 11 studies. We analyzed these two outcomes separately in the present meta-analysis.

\section{Overall Results}

Across 4 studies that evaluated client participation in treatment, the overall association with cultural competence was $r=.26(95 \% \mathrm{CI}=.05$ to $.44, p=.02 ; d=.54)$. Across 11 studies that evaluated client outcomes, the value was $r=.24(95 \% \mathrm{CI}=.10$ to $.37, p<.001 ; d=.50)$.

High heterogeneity characterized the findings of both types of outcomes analyzed: studies evaluating client participation in treatment $\left(I^{2}=80.9,95 \% \mathrm{CI}=50\right.$ to $93 ; Q_{(3)}=15.7, p=$ $.001)$ and studies evaluating treatment outcomes $\left(I^{2}=89.6,95 \% \mathrm{CI}=83\right.$ to $93 ; Q_{(10)}=96.5, p<$ .0001). The relation between therapist multicultural competence and client experiences in treatment was highly variable across studies, making it difficult to interpret the averages reported above. We therefore sought explanations for the observed variability.

\section{Moderators}

As seen in Figure 2, the effect sizes tended either to cluster around $r=0$ (indicative of no effect) or to be statistically significant (located beyond the shaded regions to the right in Figure 2), with few studies filling in the space between those extremes. We examine the data for systematic differences that could account for this unusual distribution.

As shown in Table 3, the eight effect sizes from studies measuring client perceptions of therapists' multicultural competence with client outcomes averaged $r=.38(d=.82$, a large 
effect), whereas the five effect sizes from studies using a measure of multicultural competence completed by the therapist averaged $r=.06(d=.12$, a negligible effect $)$. This difference explained a remarkable $48.2 \%$ of the variance in effect sizes in a meta-regression model that controlled for the type of outcome evaluated. Thus, this model accounted for the disparate findings shown in Figure 2.

One additional variable appeared to moderate the overall findings. The reliability coefficient of the measure of therapist multicultural competence was strongly associated with the effect size obtained in the study, $r=.49(p=.04)$. However, this finding did not remain when examined in a regression model accounting for the source of the rating (therapist or client). No other study or participant characteristic moderated the overall results.

\section{Publication Bias}

We also examined the unusual distribution of effect sizes for the possibility of publication bias influencing the results. The gap between statistically significant studies (on the right of Figure 2) and non-significant studies (in the center) suggested several "missing" studies. Nevertheless, across all studies and controlling for the type of outcome evaluated by studies, published studies yielded results of about the same magnitude as unpublished studies $(p>.20$ when testing for differences). Additional analyses, provided by Egger and Begg, as well as the trim and fill method, failed to reach significance, suggesting that the results of the meta-analysis and the shape of the data distribution were not attributable to publication status.

\section{Patient Contributions}

Mental health is embedded in cultural contexts, with the culture of the client influencing perceptions of mental health conditions and treatments (Casas et al., 2016; La Roche, 2012;

Smith, 2004). Clients engage better in therapy and experience improved outcomes when the 
treatments align with their culture. Consistent with the cultural match model (La Roche \& Lustig, 2010, 2013), client contributions attributable to culture are so salient that the more cultural adaptations to treatments, the more clinical improvement they experience.

Client age and the associated consideration of level of acculturation are also important to consider: Children and adolescents, who are more likely to be acculturated, benefit less from cultural adaptations (Huey \& Polo, 2008) than middle-aged adults and clients with low levels of acculturation. Additionally, client English proficiency likely affects treatment, with communication in the preferred language of the client being an important treatment consideration (e.g., Frandsen, Smith, Griner, \& Beecher, in press).

Client ratings of therapist multicultural competencies strongly predict their engagement and outcomes in treatment. Notably, this finding held only for client ratings, so clients' perceptions of the therapist are consequential and can be solicited by therapists.

\section{Limitations of the Research}

The first meta-analysis confirmed that cultural adaptations to mental health treatments typically prove more effective than traditional treatments when used with clients of color; however, multiple limitations characterize the current research. A key limitation is that many studies provided brief descriptions of the adaptations enacted. This limitation precludes systematic reviews like ours from accurately ascertaining the quality or specificity of adaptations implemented. Future scholarship will benefit from more detailed descriptions of specific adaptations utilized (Sidani et al., 2017). Cultural adaptations work, but we need more specific information about the underlying causal mechanisms, the reasons why they work (Smith, 2010).

The current research literature does not adequately or consistently account for several diverse groups. Most of the research on cultural adaptations in the North American literature 
involves Asian Americans and Hispanic/Latinxs; African Americans, Arab Americans, Native Americans, and Polynesian Americans remain underrepresented. Therefore, while generalizations can be made about the effectiveness of culturally adapted interventions with Asian Americans and Hispanic/Latinxs, more research is needed with other groups.

Many limitations characterize the second meta-analysis on multicultural competencies. The fact that only 15 studies met inclusion criteria suggests a strong need for additional scholarship on the topic. It appears disconcerting that so few studies evaluating clients' experiences in therapy have been conducted decades after the multicultural competencies were promoted (Sue et al., 1992). The empirical and clinical foundations of multicultural psychology will benefit from renewed interest and scholarship on this topic.

An important limitation is that therapist self-reported cultural competence did not correlate significantly $(r=.06)$ with treatment outcomes. The association between cultural competence and outcome differed dramatically depending on whether cultural competence was rated by the therapist or the client, and one has to question whether therapist self-reports of cultural competence are useful in clinical settings. Client-rated measures also involve several confounds. For example, can clients distinguish between cultural competencies and other process variables? Client evaluations may involve halo effects, wherein a positive experience with a therapist results in a globally high rating of all therapist attributes and a negative experience results in globally poor evaluations. More research is needed to address multiple potential confounds with cultural competence.

\section{Diversity Considerations}

A large body of literature has concluded that client attributes must be considered when planning and implementing mental health treatments. Our meta-analytic review evaluated only 
treatment adaptations based on client race, culture, and ethnicity; numerous other client characteristics (e.g., religious beliefs, socioeconomic status) also deserve attention (e.g., Smith, Bartz, \& Richards, 2007). Individuals espouse multiple identities at once; researchers and practitioners can account for the intersections of human diversity (Rosenthal, 2016; Smith \& Draper, 2004), such as how gender roles affect well-being among individuals with disabilities. The more a treatment is tailored to match the precise characteristics of a client, the more likely that client will engage in treatment, remain in treatment, and experience improvement as a result of treatment (Beutler et al., 2012; Castonguay \& Beutler, 2006; Smith \& Trimble, 2016). Although clinicians cannot account simultaneously for every client attribute, they can be aware of and attempt to adapt treatment to align with intersecting identities valued most by the client.

\section{Therapeutic Practices}

Cultural adaptations to mental health treatments typically prove more effective than treatment as usual with clients of color in North America. As such, these results contribute to the emerging literature on culturally adapted interventions (Chu \& Leino, 2017). Our findings show that among clients of color, their perceptions of a therapist's ability to successfully work with culture is moderately associated with their outcomes in treatment, yet these findings are susceptible to strong halo effects, wherein clients globally view therapists positively when therapy goes well. Nevertheless, individual therapist variability of client outcomes between diverse and White clients suggests the importance of cultural competencies as they pertain to improved outcomes among clients of color (Hayes et al., 2014, 2016; Imel et al., 2011). Given these findings, we urge the field to consider the following research-supported practices:

- Assess regularly patients' racial and ethnic backgrounds and their salient worldviews and race-related experiences. One cannot adapt treatments to what one does not know. 
- Improve client outcomes by aligning psychological treatment with clients' cultural backgrounds. Seek “cultural match" when planning treatments (La Roche \& Lustig, 2013).

- Incorporate multiple cultural adaptations, as they tend to be more effective than treatments with only a few cultural adaptations. The specific procedures taken to align therapy with client culture may matter less than the fact that therapists attempt to make the alignment by using several methods (Smith, 2010).

- Consider particularly cultural adaptations for adults, as they tend to prove more efficacious than with children and adolescents (Huey \& Polo, 2008), a finding likely reflecting different levels of acculturation.

- Attend to how client age and acculturation interact with potential cultural adaptations to treatments.

- Conduct psychotherapy in the client's preferred language or utilize an interpreter with appropriate training and cultural knowledge (APA, 1993; Frandsen et al., in press).

- Adapt therapy to a specific cultural group, rather than a culturally mixed group of clients. The more culturally specific the treatment, the more effective it will probably prove.

- Handle cultural issues for clients of color in a sensitive and humble way. How clients perceive psychotherapists is strongly related with their treatment outcomes.

- Assume that therapists' own self-evaluations regarding multicultural competence do not align with the perspectives of clients of color. To overcome that gap, therapists can actively seek to learn each client's perspectives.

- Add questions about cultural competence to routinely administered questionnaires (e.g., quality of alliance, treatment progress). That is, privilege the client's experience of whether the psychotherapist or treatment accords and resonates with their cultural identities. 


\section{Select References and Recommended Readings}

Benish, S. G., Quintana, S., \& Wampold, B. E. (2011). Culturally adapted psychotherapy and the legitimacy of myth: A direct-comparison meta-analysis. Journal of Counseling Psychology, 58(3), 279-289. https://doi.org/10.1037/a0023626

Bernal, G., Bonilla, J., \& Bellido, C. (1995). Ecological validity and cultural sensitivity for outcome research: Issues for the cultural adaptation and development of psychosocial treatments with Hispanics. Journal of Abnormal Child Psychology, 23(1), 67-82.

Cabral, R. R., \& Smith, T. B. (2011). Racial/ethnic matching of clients and therapists in mental health services: A meta-analytic review of preferences, perceptions, and outcomes. Journal of Counseling Psychology, 58(4), 537-554. https://doi.org/10.1037/a0025266

Cardemil, E. V. (2010). Cultural adaptations to empirically supported treatments: A research agenda. The Scientific Review of Mental Health Practice, 7(2), 8-21.

Degnan, A., Baker, S., Edge, D., Nottidge, W., Noke, M., Press, C. J., ... \& Drake, R. J. (2017). The nature and efficacy of culturally-adapted psychosocial interventions for schizophrenia: a systematic review and meta-analysis. Psychological Medicine, 1-14.

Domenech Rodríguez, M. M., \& Bernal, G. (2012). Cultural adaptations: Tools for evidencebased practice with diverse populations. Washington, DC: APA Press.

Dunn, T., Smith, T. B., \& Montoya, J. (2006). Multicultural competency instrumentation: A review and analysis of reliability generalization. Journal of Counseling and Development, $84,471-482$.

Frandsen, C., Smith, T. B., Griner, D., \& Beecher, M. (In press). Beyond English Only: Addressing language interpretation in professional psychology training. Training and Education in Professional Psychology. 
Griner, D., \& Smith, T. B. (2006). Culturally adapted mental health intervention: A metaanalytic review. Psychotherapy: Theory, Research, Practice, Training, 43(4), 531-548.

Hall, G. C. N., Ibaraki, A. Y., Huang, E. R., Marti, C. N., \& Stice, E. (2016). A meta-analysis of cultural adaptations of psychological interventions. Behavior Therapy, 47, 993-1014.

Huey, S. J., \& Polo, A. J. (2008). Evidence-based psychosocial treatments for ethnic minority youth. Journal of Clinical Child \& Adolescent Psychology, 37(1), 262-301.

Imel, Z. E., Baldwin, S., Atkins, D. C., Owen, J., Baardseth, T., \& Wampold, B. E. (2011). Racial/ethnic disparities in therapist effectiveness: A conceptualization and initial study of cultural competence. Journal of Counseling Psychology, 58, 290-298.

La Roche, M. J. (2012). Cultural psychotherapy: Theory, methods, \& practice. Thousand : Sage. Smith, T. B. (Ed.). (2004). Practicing multiculturalism: Affirming. Boston: Allyn \& Bacon. Smith, T. B. (2010). Culturally congruent practices in counseling and psychotherapy: A review of research. In J. G. Ponterotto, J. M. Casas, L. A. Suzuki \& C. M. Alexander (Eds.), Handbook of multicultural counseling (3rd ed., pp. 439-450). Thousand Oaks, CA: Sage.

Smith, T. B., Bartz, J., \& Richards, P. S. (2007). Outcomes of religious and spiritual adaptations to psychotherapy: A meta-analytic review. Psychotherapy Research, 17(6), 643-655.

Smith, T. B., Domenech Rodríguez, M. D., \& Bernal, G. (2011). Culture. Journal of Clinical Psychology, 67(2), 166-175.

Smith, T. B., \& Trimble, J. E. (2016). Foundations of multicultural psychology: Research to inform effective practice. Washington, DC: American Psychological Association.

Sue, D. W., Arredondo, P., \& McDavis, R. J. (1992). Multicultural counseling competencies and standards: A call to the profession. Journal of Counseling \& Development, 70, 477-486.

Tao, K. W., Owen, J., Pace, B. T., \& Imel, Z. E. (2015). A meta-analysis of multicultural competencies and psychotherapy process and outcome. Journal of Counseling Psychology, 62(3), 337. 
Figure 1. Contour-enhanced funnel plot of effect sizes (Cohen's $d$ ) by standard error for 99 studies of culturally adapted treatments.

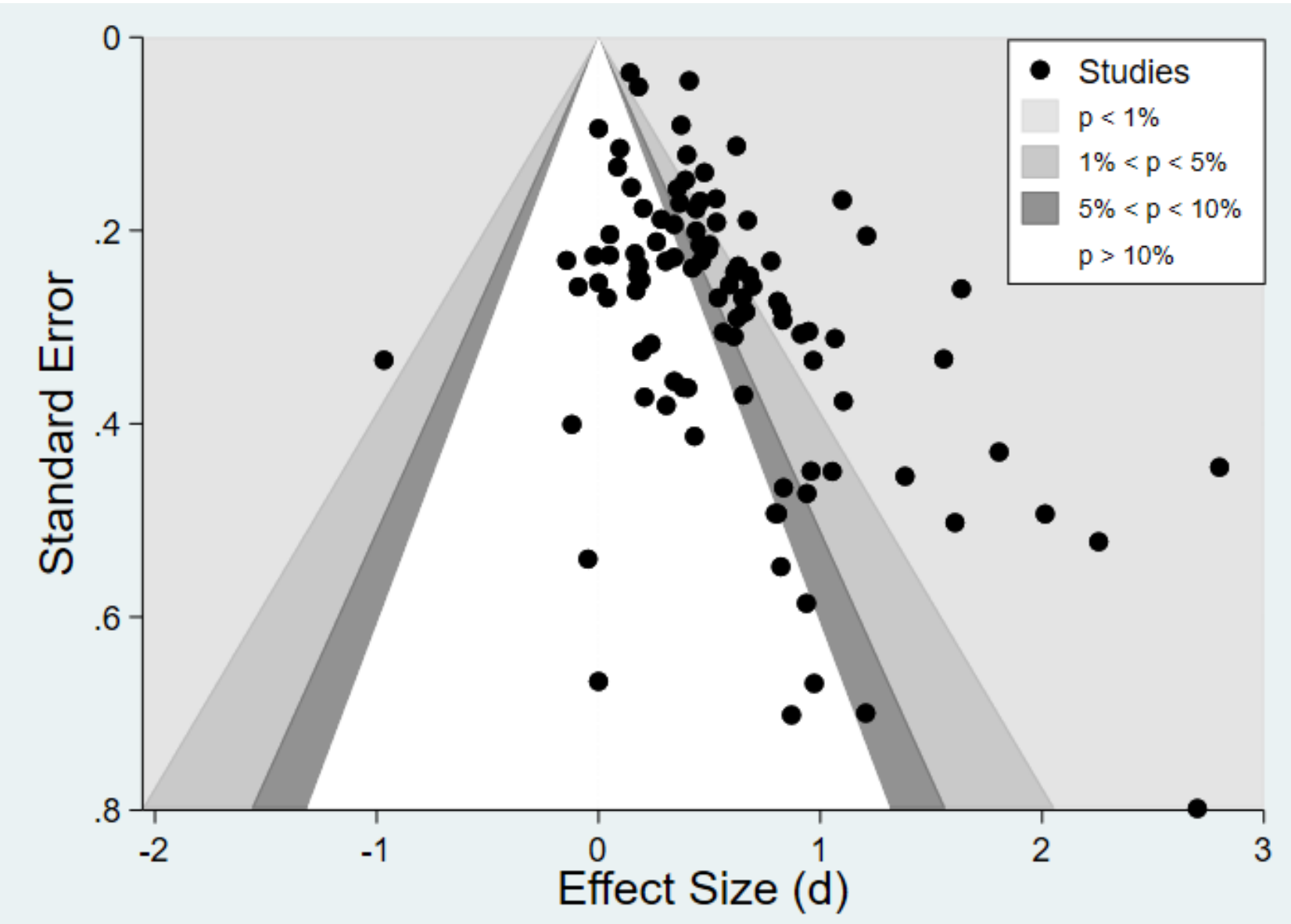

Note: This graph shows the distribution of effect sizes as a function of the number of participants in the study (operationalized as standard error). 
Figure 2. Contour-enhanced funnel plot of effect sizes (Pearson $r$ ) by standard error for 15 correlational studies of therapist multicultural competence and client treatment outcomes.

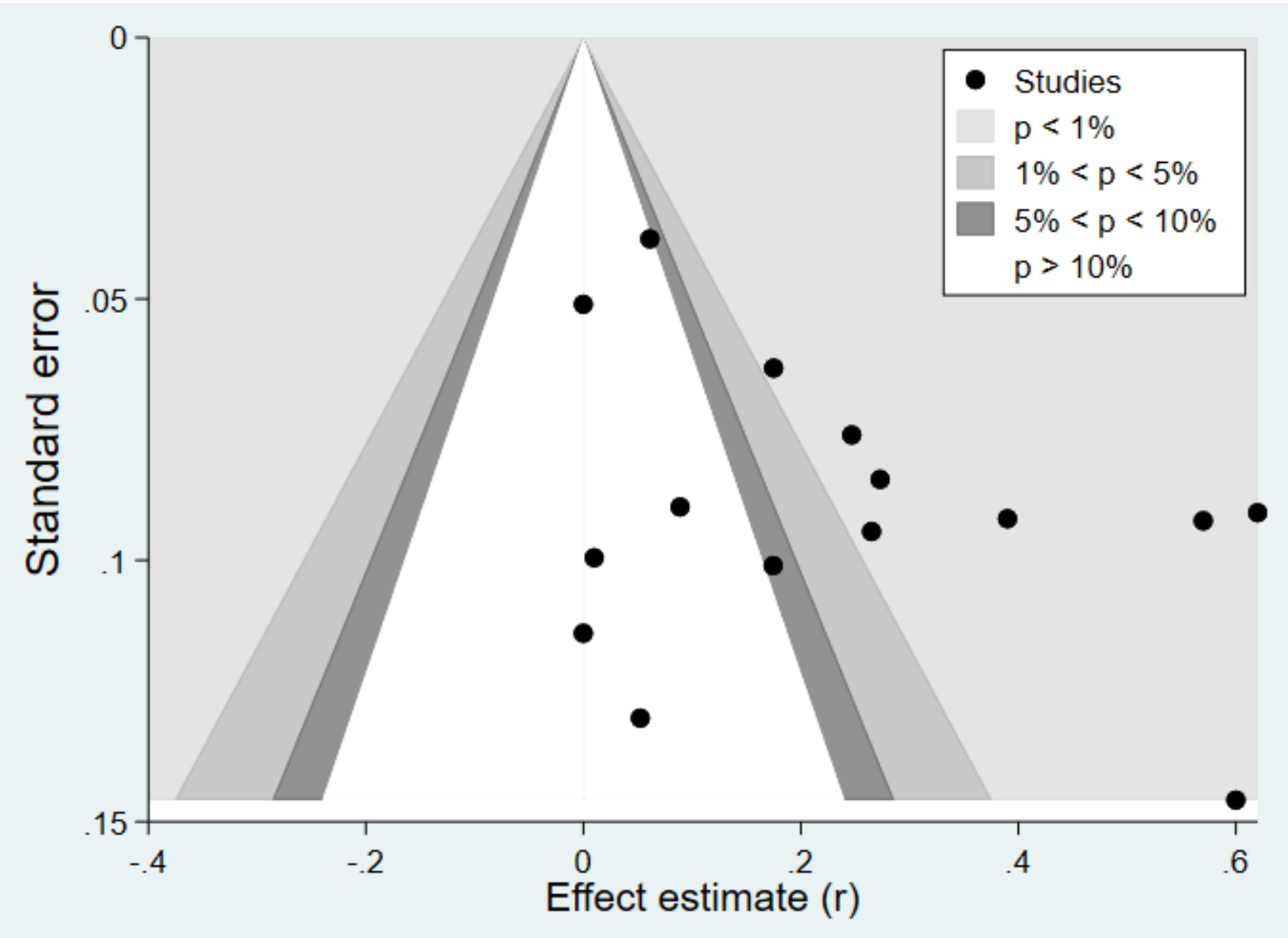

Note: This graph shows the distribution of effect sizes as a function of the number of participants in the study (operationalized as standard error). The results are highly scattered. 
Table 1

Moderator Analyses for the Studies of Cultural Adaptations to Mental Health Treatments (Metaanalysis 1)

\begin{tabular}{llllll}
\hline Variable & $Q$ & $p$ & $k$ & $d$ & $95 \% \mathrm{CI}$
\end{tabular}

\section{Study Characteristics}

Research Design

Quasi-experimental

Experimental

Control Group Type

No treatment (e.g., waiting list)

Treatment as usual

Outcome Measures ${ }^{a}$
$9.1 \quad .003$
23

76

0.5

.47
General/multi-dimensional wellbeing

$15.0 \quad .002$

General/multi-dimensional mental health symptoms

$\begin{array}{lll}45 & .53 & {[.41, .64]} \\ 53 & .47 & {[.37, .57]}\end{array}$

Specific mental health symptoms (e.g., depression)

18

.23

$[.06, .41]$

12

.26

$[.04, .48]$

43

.55

$[.43, .67]$

Specific behavioral outcomes (e.g., aggression)

46

.58

$[.46, .70]$

Treatment Characteristics

Type of Treatment ${ }^{b}$

$6.3 \quad .01$

Mental health treatments

$[.31, .52]$

Prevent programs targeting problem behaviors

12

.68

$[.50, .86]$

Treatment Modality

$1.2 \quad .75$

Individual

18

.48

$[.30, .67]$

Group

46

.48

$[.36, .59]$

Family

14

.63

$[.38, .87]$

Combined individual/group

11

.50

$[.27, .73]$

Adaptations for Client Language ${ }^{c}$

$4.7 \quad .03$

No

17

.35

$[.16, .54]$

Yes

52

.59

$[.48, .70]$

Measurement Language Translation ${ }^{c}$

$18.2<.0001$

No

23

.28

$[.15, .42]$

Yes

46

.66

$[.55, .76]$ 
Goals Based on Cultural Values

$4.9 \quad .03$

No

85

.46

$[.38, .54]$

Yes

14

.69

$[.50, .88]$

Use of Cultural Metaphors

$5.3 \quad .02$

No

57

.43

$[.33, .52]$

Yes

42

.61

$[.49, .73]$

Adaptations to Treatment Methods

$4.3 \quad .04$

No

51

.42

$[.32, .53]$

Yes

48

.58

$[.47, .69]$

\section{Participant Characteristics}

Gender of Client

$0.3 \quad .57$

Female

22

Male

6

.53

$[.35, .70]$

.42

$[.08, .75]$

Ethnicity of Client

$7.6 \quad .06$

African American

$23 \quad .50 \quad[.33, .67]$

Hispanic/Latinx American

$44 \quad .52 \quad[.41, .64]$

Asian American

$18 \quad .67 \quad[.47, .88]$

Native American

$5 \quad .14 \quad[-.18, .46]$

Clients All Same Ethnicity

$6.3 \quad .01$

No

$\begin{array}{rrr}9 & .29 & {[.05, .54]} \\ 67 & .63 & {[.53, .74]}\end{array}$

Yes

$4.0 \quad .04$

Sample Type

Community/at-risk samples

Clinical populations

Treatment by Age Grouping

At-risk samples under age 18

33

.36

$[.23, .49]$

Clinical samples over age 24

20

.70

$[.52, .88]$

Note. $Q=\mathrm{Q}$-value for variance between groups. $p=$ significance of between group differences. $k$ $=$ number of studies. $d=$ Cohen's $d$, random effects weighted effect size. ${ }^{\text {a }}$ This analysis compared all outcome measures within studies. ${ }^{\mathrm{b}}$ Culturally adapted treatments compared with treatment as usual. ${ }^{\mathrm{c}}$ Among populations likely speaking English as a second language. 
Table 2

Random Effects Regression Weights for Study Characteristics Associated with Effect Sizes from Studies of Cultural Adaptations to Mental Health Treatments (Meta-analysis 1).

\begin{tabular}{|c|c|c|c|}
\hline Variable & $R^{2}$ & $\beta$ & $p$ \\
\hline & $35.4^{* * *}$ & & \\
\hline Estimate of publication bias ${ }^{\text {a }}$ & & .256 & .002 \\
\hline Assessments of specific symptoms ${ }^{b}$ & & .246 & .003 \\
\hline Random assignment to treatment type & & .214 & .01 \\
\hline Sum of cultural adaptations ${ }^{c}$ & & .175 & .04 \\
\hline Average client age & & .177 & .09 \\
\hline Clinical sample vs. at-risk or community sample & & .150 & .13 \\
\hline Treatment for problematic behavior ${ }^{\mathrm{d}}$ & & .142 & .11 \\
\hline Year of study publication & & .083 & .37 \\
\hline Client racial homogeneity within studies ${ }^{\mathrm{e}}$ & & .070 & .41 \\
\hline
\end{tabular}

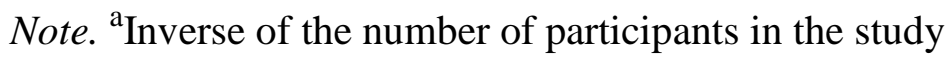

${ }^{\mathrm{b}}$ Contrast of symptom-specific measures of mental health and pro-social behaviors with multidimensional assessments or global well-being/mental health

${ }^{\mathrm{c}}$ Sum of the eight indicators of the ecological model (Bernal et al., 1995)

${ }^{\mathrm{d}}$ Contrast of treatments for problematic behaviors with treatments for mental health

${ }^{e}$ Contrast of studies in which all participants were of the same race with studies in which participants' race varied

$* * * p<.0001$ 
Table 3

Moderator Analyses for the Studies of Multicultural Competence (Meta-analysis 2).

Variable

$Q$

b p

k

$r$

$95 \% \mathrm{CI}$

\section{Between Study Differences}

Source of Competence Evaluation

12.5

.0004

Therapist (self-report)

$5 \quad .06 \quad[-.08, .20]$

Clients

$8 \quad .38 \quad[.27, .48]$

Published

0.5

.47

No

$6 \quad .19 \quad[.00, .37]$

Yes

0.2

$9 \quad .28$

$[.13, .41]$

Clients All Same Ethnicity

.67

No

4

.29

$[.06, .52]$

Yes

11

.23

$[.10, .37]$

\section{Correlations with Effect Size}

Year of Study

Average Participant Age

Gender (\% females)

$\%$ African Americans

$\%$ Asian Americans

$\%$ Hispanic/Latinx Americans

Multicultural competence measure reliability

$.29 \quad .25 \quad 15$

$\begin{array}{lll}-.15 & .67 \quad 10\end{array}$

$\begin{array}{lll}-.11 & .68 & 14\end{array}$

$\begin{array}{lll}.16 & .53 \quad 15\end{array}$

$\begin{array}{lll}-.07 & .79 & 15\end{array}$

$\begin{array}{lll}-.29 & .25 \quad 15\end{array}$

$.49 \quad .04 \quad 13$

Note. $Q=\mathrm{Q}$-value for variance between groups. $b=$ standardized coefficient for the association with effect size. $p=$ significance of between group differences. $k=$ number of studies. $r=$ random effects weighted correlation coefficient. 